François Cachat • Alina Bogaru • Jean-Leopold Micheli • Domenico Lepori • Jean-Pierre Guignard

\title{
Severe hypertension and massive proteinuria in a newborn with renal artery stenosis
}

Received: 23 June 2003 / Revised: 18 November 2003 / Accepted: 18 November 2003 / Published online: 21 February 2004 (C) IPNA 2004

\begin{abstract}
Renal vein thrombosis and the congenital nephrotic syndrome have been associated with nephrotic-range proteinuria/nephrotic syndrome and hypertension in the newborn period. We describe a newborn with severe hypertension and proteinuria secondary to unilateral renal artery stenosis. Proteinuria completely disappeared with blood pressure control (with sodium nitroprusside and an angiotensin-converting enzyme inhibitor). Although renin was not measured, we speculate that proteinuria might have been induced by a high renin state, and was controlled by the angiotensin-converting enzyme inhibitor.
\end{abstract}

Keywords Renal artery stenosis - Hypertension . Proteinuria $\cdot$ Nephrotic syndrome $\cdot$ Newborn $\cdot$ Renin

\section{Introduction}

The simultaneous occurrence of hypertension and proteinuria/nephrotic syndrome in a newborn raises the differential diagnosis of a congenital nephrotic syndrome or a renal vein thrombosis (RVT). Renal artery stenosis (RAS) has been associated with proteinuria or even nephrotic syndrome, mainly in adult patients. Recently, Lang and Gowrishankar [1] described a young child with RAS and nephrotic syndrome. We wish to further expand

\footnotetext{
F. Cachat $(\bowtie) \cdot$ A. Bogaru - J.-P. Guignard

Department of Pediatrics, Division of Pediatric Nephrology, University Hospital, Lausanne, Switzerland

e-mail: Francois.Cachat@hospvd.ch

Tel.: +41-21-3141111

Fax: +41-21-3143626

J.-L. Micheli

Division of Neonatology,

University Hospital, Lausanne, Switzerland

D. Lepori

Department of Radiology, Division of Pediatric Radiology, University Hospital, Lausanne, Switzerland
}

the rare clinical association of RAS, hypertension, and nephrotic-range proteinuria in a newborn baby.

\section{Case report}

The female patient was delivered vaginally at 42 weeks of gestation, after an uneventful pregnancy. The 24-year-old mother had no diabetes and was on no medications. The birth weight was $3,485 \mathrm{~g}$ (50th percentile) and length $50 \mathrm{~cm}$ (10th-50th percentile). Apgar scores were 7, 9, and 9 at 1,5 , and $10 \mathrm{~min}$, respectively. Initial blood pressure values (10 h after birth) were high at $85 /$ $50 \mathrm{mmHg}$ [2]. Because of mild tachypnea, the child was admitted to a neonatal division and closely monitored. On day 3 of life, blood pressure was $100-105 / 80 \mathrm{mmHg}$, and she developed nephrotic-range proteinuria and microscopic hematuria. Laboratory results included plasma creatinine $76 \mu \mathrm{mol} / 1$, blood urea nitrogen $3.9 \mathrm{mmol} / \mathrm{l}$, and albumin $33 \mathrm{~g} / \mathrm{l}$. The urinary protein/creatinine ratio was $5,807 \mathrm{~g} / \mathrm{mol}(66 \mathrm{~g} / \mathrm{l})$. Urinalysis revealed $0-1$ white blood cells/ high-power field (HPF), 10-15 red blood cells/HPF, and no casts. Renal ultrasonography showed two normal-sized kidneys with normal arterial and venous blood flow and no signs of venous thrombosis. The infant's condition deteriorated and she was transferred to our hospital. On admission (day 5 of life), she was hypertensive $(115 / 86 \mathrm{mmHg})$ and in heart failure. There was an enlarged liver and generalized edema. Laboratory investigations confirmed the proteinuria $(1,176 \mathrm{~g} / \mathrm{mol})$, with mild hypoalbuminemia (30 g/l), no electrolyte abnormalities, creatinine $96 \mu \mathrm{mol} / \mathrm{l}$, serum glutamic oxaloacetic transaminase $66 \mathrm{U} / \mathrm{l}$, and serum glutamic pyruvic transaminase $110 \mathrm{U} / \mathrm{l}$. Echocardiography showed a slight hypertrophied and dilated left ventricle, with an ejection fraction of $37 \%$. There was a slight mitral and aortic insufficiency. Because of failure of captopril, furosemide, and hydralazine to adequately control the blood pressure, sodium nitroprusside was added. The blood pressure response was immediate (Fig. 1). Oral labetalol was added on day 9 of life, in order to wean the infant off sodium nitroprusside. Two weeks later, the infant's blood pressure was controlled with oral labetalol (16 $\mathrm{mg}$ three times a day) and captopril $(0.1 \mathrm{mg}$ twice daily). Proteinuria decreased and then disappeared as soon as the blood pressure normalized (Fig. 1). At discharge, there was no proteinuria and no hematuria. On day 8 of life, 3 days after a single dose of furosemide, the patient also presented with hyponatremia $(128 \mathrm{mmol} / \mathrm{l})$ secondary to renal salt wasting (urine sodium $92 \mathrm{mmol} / \mathrm{l}$ ). Hyponatremia resolved spontaneously, without sodium supplementation. Duplex Doppler sonography on day 9 of life showed a small right kidney with a decreased arterial signal, but normal venous flow; the left kidney was normal. Magnetic resonance angiography revealed a stenotic/ hypoplastic right renal artery and a normal left renal artery (Fig. 2). 
Fig. 1 Follow-up of proteinuria and blood pressure values $(S B P$ systolic blood pressure, $D B P$ diastolic blood pressure)
Variation of proteinuria with blood pressure control

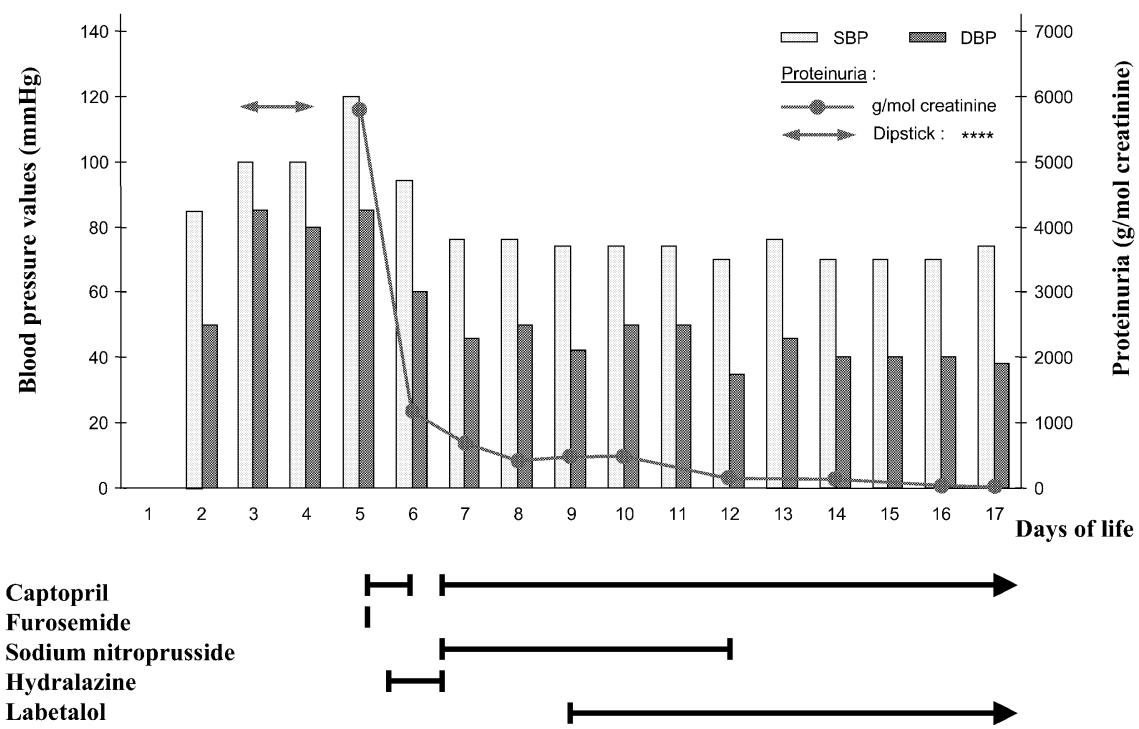

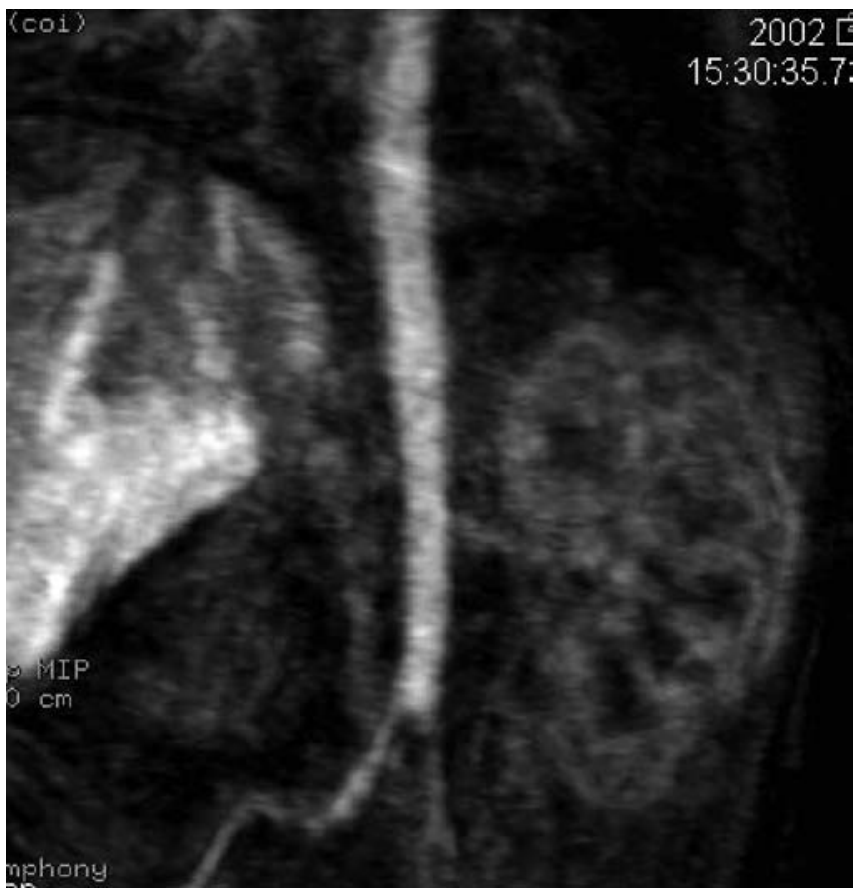

Fig. 2 Abdominal aortic magnetic resonance angiography. The left renal artery and left kidney are clearly delineated. The right renal artery and kidney are not visible

Renal scan (Mag3) performed on day 10 of life showed no activity on the right side and a compensatory hyperactivity on the left side. At discharge (day 18 of life), the patient was normotensive, with a creatinine of $56 \mu \mathrm{mol} / \mathrm{l}$ and sodium of $135 \mathrm{mmol} / \mathrm{l}$. Eight months later, she is on enalapril $0.6 \mathrm{mg}$ twice daily, her blood pressure is normal $(90 / 50 \mathrm{mmHg}$ ), and she has no proteinuria or hematuria. Follow-up renal ultrasonography, a year later, shows a small atrophic right kidney $(<5$ th percentile) with a hypertrophied left kidney (95th percentile). Echocardiography performed 3 months after discharge is also normal, with complete regression of the left ventricular failure. A repeat renal scan confirmed the total absence of perfusion of the right kidney, and showed a well-functioning left kidney, with some mild irregularities and a heterogeneous distribution of the nuclear tracer.

\section{Discussion}

The differential diagnosis of severe hypertension in a newborn baby includes acquired or congenital renal vascular diseases (RAS, renal artery hypo(dys)plasia, thromboembolic events), renal parenchymal/glomerular disorders, obstructive uropathies, coarctation of the aorta, or drug-induced hypertension [3]. Amongst those causes, only RVT, congenital nephrotic syndrome (of any etiology), or RAS have been associated with nephrotic-range proteinuria or even frank nephrotic syndrome. Although RVT can rarely induce a nephrotic syndrome $[4,5]$, it is mostly a direct consequence of hemoconcentration and chronic urinary loss of anti-thrombin III secondary to a primary long-standing nephrotic syndrome. RVT was excluded in our patient (no thrombopenia, no macroscopic hematuria, normal renal venous blood flow, no enlarged hyperechogenic kidneys). RAS has also been associated with mild-to-severe proteinuria [6] or even nephrotic syndrome $[1,7]$. The occurrence of proteinuria after renal artery occlusion is thought to be secondary either to the high renin state and tissue ischemia in the occluded kidney or to an excessive blood flow in the contralateral healthy kidney, or both [6]. Focal and segmental glomerulosclerosis of the healthy contralateral kidney has also been described in patients with longstanding RAS [8]. Proteinuria in that case is secondary to chronic hyperfiltration of the remaining nephrons.

Although plasma renin activity was not measured, we suggest that the nephrotic-range proteinuria and the hypertension in our patient were induced or exacerbated by the exaggerated renin production in the ischemic right kidney. 
Proteinuria decreased and then disappeared with the administration of an angiotensin-converting enzyme inhibitor. Renin increases angiotensin II, which has been shown to modulate glomerular capillary permselectivity in experimental animals [9]. Our patient also presented with transient renal sodium loss. Renal salt wasting has also been reported in malignant hypertension [10]. The mechanism of sodium loss is not completely understood. In animal experiments, an acute increase in the arterial perfusion pressure in the isolated kidney of the dog led to an increase in urine flow and sodium excretion, whereas the glomerular filtration rate and renal blood flow were unchanged [11]. The persistence of hypertension, more than a year after the initial event, might be explained by persistent renin secretion in the ipsilateral kidney, or by the contralateral kidney, which showed numerous irregularities on renal scan, corresponding probably to renal scars.

In summary, we describe a newborn baby with hypertension and nephrotic-range proteinuria, secondary to RAS, which responded well to an angiotensin-converting enzyme inhibitor. We speculate that an excessively stimulated renin-angiotensin-aldosterone system might have been responsible for the heavy proteinuria, through altered permeability of the glomerular capillaries. RVT, congenital nephrotic syndrome, and likewise RAS should be ruled out in a newborn presenting with severe hypertension and proteinuria.

\section{References}

1. Lang ME, Gowrishankar M (2003) Renal artery stenosis and nephrotic syndrome: a rare combination in an infant. Pediatr Nephrol 18:276-279

2. Versmold HT, Kitterman JA, Phibbs RH, Gregory GA, Tooley WH (1981) Aortic blood pressure during the first 12 hours of life in infants with birth weight 610 to 4220 grams. Pediatrics 67:607-613

3. Guignard JP, Gouyon JB, Adelman RD (1989) Arterial hypertension in the newborn infant. Biol Neonate 55:77-83

4. Cohn LH, Lee JL, Hopper J, Najarian JS (1968) The treatment of bilateral renal vein thrombosis and nephrotic syndrome. Surgery 64:387-396

5. Janower ML (1965) Nephrotic syndrome secondary to renal vein thrombosis. The value of inferior vena cavography. Am J Roentgenol Radium Ther Nucl Med 95:330-334

6. Rossignol P, Chatellier G, Azizi M, Plouin PF (2002) Proteinuria in renal artery occlusion is related to active renin concentration and contralateral kidney size. J Hypertens 20:139-144

7. Pasternack A, Eklund J, Krohn K (1967) Renal artery stenosis and the nephrotic syndrome. Acta Med Scand 181:265-268

8. Ubara Y, Hara S, Katori H, Yamada A, Morii H (1997) Renovascular hypertension may cause nephrotic range proteinuria and focal glomerulosclerosis in contralateral kidney. Clin Nephrol 48:220-223

9. Lapinski R, Perico N, Remuzzi A, Sangalli F, Benigni A, Remuzzi G (1996) Angiotensin II modulates glomerular capillary permselectivity in rat isolated perfused kidneys. J Am Soc Nephrol 7:653-660

10. Blanc F, Bensman A, Baudon JJ (1991) Renovascular hypertension: a rare cause of neonatal salt loss. Pediatr Nephrol 5:304-306

11. Barraclough MA (1966) Sodium and water depletion with the acute malignant hypertension. Am J Med 40:265-272 